\title{
Asymptotic Behavior of Oscillatory Solutions of First Order Delay Differential Equations of Unstable Type
}

\author{
By
}

\author{
Tateo BABA
}

(Obirin High School, Japan)

\begin{abstract}
In this paper, we study the asymptotic behavior of solutions of the first order delay differential equations of unstable type. A new sufficient condition is given under which every oscillatory solution of the delay equation of unstable type tends to zero asymptotically.

Key Words and Phrases. Delay differential equation, Oscillatory solution, Asymptotic behavior, Unstable type.

2010 Mathematics Subject Classification Numbers. 34K11, 34K25.
\end{abstract}

\section{Introduction}

We consider the following first order delay differential equation of unstable type

$$
x^{\prime}(t)=q(t) x(\sigma(t)), \quad t \geq t_{0},
$$

where $q:\left[t_{0}, \infty\right) \rightarrow[0, \infty)$ is a piecewise continuous function, $\sigma:\left[t_{0}, \infty\right) \rightarrow$ $(-\infty, \infty)$ is a continuous, monotone increasing function such that $\sigma(t) \leq t$ and $\sigma(t) \rightarrow \infty$ as $t \rightarrow \infty$.

The purpose of this paper is to give a new sufficient condition under which every oscillatory solution of $(1.1)$ tends to zero as $t \rightarrow \infty$. In this paper, a solution of (1.1) is called oscillatory if it has arbitrarily large zeros.

In [2], Györi studied a general delay differential equation of unstable type which includes (1.1) as a special case and obtained a sufficient condition

$$
\limsup _{t \rightarrow \infty} \int_{\sigma(t)}^{t} q(s) d s<2,
$$

when $\tau(t)=t-\sigma(t)$ is bounded, under which every oscillatory solution of (1.1) tends to zero as $t \rightarrow \infty$. Moreover, for a special case of (1.1),

$$
x^{\prime}(t)=q x(t-\tau), \quad t \geq t_{0},
$$

where $q$ and $\tau$ are positive constants, Györi ([2, Theorem 4.2]) proved that $q \tau\left(=\int_{t-\tau}^{t} q d s\right)<3 \pi / 2$ is a necessary and sufficient condition for every oscillatory solution of (1.3) to tend to zero as $t \rightarrow \infty$. 
Recently, Philos et al. [8] studied certain delay differential equations of unstable type and derived (1.2) as a consequence of general results. As they pointed out, the gap between the bound 2 in (1.2) and the bound $3 \pi / 2$ still remains.

The main purpose of the present paper is to narrow this gap by showing that the bound 2 can be improved to $5 / 2$. In fact, we will show (Corollary 2 in section 2) that

$$
\limsup _{t \rightarrow \infty} \int_{\sigma(t)}^{t} q(s) d s<\frac{5}{2}
$$

is a sufficient condition for every oscillatory solution of (1.1) to tend to zero as $t \rightarrow \infty$ even when $\tau(t)=t-\sigma(t)$ may be unbounded.

In section 3 , we will study the following equation:

$$
x^{\prime}(t)=q(t) x(\sigma(t))+f(t), \quad t \geq t_{0},
$$

where $f(t)$ is a continuous function of certain type, and it will be shown that (1.4) is a sufficient condition for every oscillatory solution of (1.5) to tend to zero as $t \rightarrow \infty$. For a special case of (1.5),

$$
x^{\prime}(t)=q x(t-\tau)+k e^{-\lambda t}, \quad t \geq t_{0},
$$

where $k$ is a constant, $q, \tau$ and $\lambda$ are positive constants, we will prove that $q \tau<3 \pi / 2$ is a necessary and sufficient condition for every oscillatory solution of (1.6) to tend to zero as $t \rightarrow \infty$.

In section 4 , we will give a simple example which shows that

$$
\limsup _{t \rightarrow \infty} \int_{\sigma(t)}^{t} q(s) d s<\frac{15}{4}
$$

is a necessary condition for every oscillatory solution of (1.1) to tend to zero as $t \rightarrow \infty$.

Concerning the asymptotic behavior of solutions of delay differential equations, we refer to $[1,3-6]$. We also refer to $[7,9,10]$; the methods used in these papers will be useful to prove our Theorem 1.

Throughout this paper we use the notation $\sigma^{2}(t)=\sigma(\sigma(t)), \sigma^{3}(t)=\sigma\left(\sigma^{2}(t)\right)$ and $\sigma^{-2}(t)=\sigma^{-1}\left(\sigma^{-1}(t)\right)$, where $\sigma^{-1}$ is the inverse function of $\sigma$. And we also use

$$
Q(T):=\sup _{t \geq T} \int_{\sigma(t)}^{t} q(s) d s
$$


for $T \geq \sigma^{-1}\left(t_{0}\right)$ and

$$
Q_{\infty}:=\lim _{T \rightarrow \infty} Q(T)=\limsup _{t \rightarrow \infty} \int_{\sigma(t)}^{t} q(s) d s .
$$

\section{Main results}

Lemma 1. Let $x(t)$ be a solution of (1.1) and let $\sigma^{-1}\left(t_{0}\right)<t_{1}<t_{2}$. If $x(t) \neq 0$ for $t \in\left(t_{1}, t_{2}\right)$ and $x\left(t_{2}\right)=0$, then $t_{1} \geq \sigma\left(t_{2}\right)$.

Proof. Assume that the conclusion is not true, that is, $t_{1}<\sigma\left(t_{2}\right)$. Without loss of generality, we may assume $x(t)>0$ for $t \in\left(t_{1}, t_{2}\right)$. There exists an $\omega$ satisfying $t_{1}<\sigma(\omega) \leq \omega<t_{2}$. Then $x^{\prime}(t)=q(t) x(\sigma(t)) \geq 0$ for $t \in\left[\omega, t_{2}\right]$, which implies $x\left(t_{2}\right) \geq x(\omega)>0$. This is a contradiction. The proof is complete.

Lemma 2. Given $\delta>0$, there exists a continuous increasing function $h:\left[t_{0}, \infty\right) \rightarrow[0, \infty)$ such that

$$
h(t)-h(\sigma(t)) \leq \delta, \quad t \geq \sigma^{-1}\left(t_{0}\right) .
$$

Proof. Choose a sequence $\left\{T_{n}\right\}_{n=0,1,2, \ldots}$ such that $T_{0}=t_{0}$ and for $n=0,1,2, \ldots$,

$$
\begin{cases}T_{n+1}=T_{n}+1 & \text { if } \sigma^{-1}\left(T_{n}\right)-T_{n}<1 \\ T_{n+1}=\sigma^{-1}\left(T_{n}\right) & \text { if } \sigma^{-1}\left(T_{n}\right)-T_{n} \geq 1 .\end{cases}
$$

Then $T_{n} \leq \sigma\left(T_{n+1}\right), T_{n+1}-T_{n} \geq 1$ for all $n$ and $\lim _{n \rightarrow \infty} T_{n}=\infty$. Let us define

$$
h(t)=\frac{\delta}{2}\left(\frac{t-T_{n}}{T_{n+1}-T_{n}}+n\right) \quad \text { for } t \in\left[T_{n}, T_{n+1}\right)
$$

for $n=0,1,2, \ldots$ We see that $h\left(T_{n}\right)=n \delta / 2$ for all $n$ and $h(t)$ is a continuous increasing function on $\left[t_{0}, \infty\right)$. For $t \in\left[T_{n}, T_{n+1}\right), n=1,2,3, \ldots$,

$$
h(t)<h\left(T_{n+1}\right) \quad \text { and } \quad h(\sigma(t)) \geq h\left(\sigma\left(T_{n}\right)\right) \geq h\left(T_{n-1}\right),
$$

which implies

$$
h(t)-h(\sigma(t)) \leq h\left(T_{n+1}\right)-h\left(T_{n-1}\right)=\delta .
$$

Therefore (2.1) holds for $t \geq T_{1}$. When $\sigma^{-1}\left(t_{0}\right)<T_{1}$, it follows that $h(t)-h(\sigma(t)) \leq \delta$ for $t \in\left[\sigma^{-1}\left(t_{0}\right), T_{1}\right)$, which means that $(2.1)$ holds. Hence the proof is complete.

Theorem 1. Let $h:\left[t_{0}, \infty\right) \rightarrow[0, \infty)$ be a continuous increasing function satisfying (2.1) for some $\delta>0$. If $Q(T) \leq 5 / 2$ for some $T \geq \sigma^{-1}\left(t_{0}\right)$, then 
for any oscillatory solution $x(t)$ of (1.1), there exists a $K=K(\beta, h, x)>0$ such that

$$
|x(t)|<K e^{-\beta h(t)}, \quad t \geq t_{0},
$$

where

$$
\begin{cases}\beta=\frac{2}{3 \delta} \log \frac{2}{Q(T)} & \text { if } Q(T)<1 \\ \beta=\frac{1}{3 \delta} \log \frac{4}{2 Q(T)-1} & \text { if } 1 \leq Q(T) \leq \frac{5}{2}\end{cases}
$$

Proof. Since $x(t)$ is an oscillatory solution of (1.1), there exists a sufficiently large number $w_{0}>\sigma^{-2}(T)$ such that $x\left(w_{0}\right)=0$. We will show that (2.2) holds for a positive constant $K$ such that

$$
K>\max _{t_{0} \leq t \leq w_{0}} e^{\beta h(t)}|x(t)| .
$$

Assume that (2.2) does not hold. Then there exists a $\xi>w_{0}$ such that

$$
|x(t)|<K e^{-\beta h(t)} \quad \text { for } t \in\left[t_{0}, \xi\right) \quad \text { and } \quad|x(\xi)|=K e^{-\beta h(\xi)} .
$$

Since $x(t)$ is oscillatory and $\xi>w_{0}$, we can define two real numbers $t_{1}$ and $t_{2}$ by

$$
t_{1}=\sup \{t: t<\xi, x(t)=0\},
$$

and

$$
t_{2}=\inf \{t: t>\xi, x(t)=0\} .
$$

We see that $x\left(t_{1}\right)=x\left(t_{2}\right)=0, w_{0} \leq t_{1}<\xi<t_{2}$ and $x(t) \neq 0$ for $t \in\left(t_{1}, t_{2}\right)$. Lemma 1 leads to $t_{1} \geq \sigma\left(t_{2}\right)$. Since $\sigma(u)$ is monotone increasing, $t_{1} \geq \sigma(u)$ for $u \in\left[t_{1}, t_{2}\right]$. Then

$$
\begin{aligned}
|x(\sigma(u))| & =\left|x\left(t_{1}\right)-x(\sigma(u))\right| \\
& =\left|\int_{\sigma(u)}^{t_{1}} x^{\prime}(s) d s\right| \\
& =\left|\int_{\sigma(u)}^{t_{1}} q(s) x(\sigma(s)) d s\right|,
\end{aligned}
$$

which implies that for $u \in\left[t_{1}, t_{2}\right]$,

$$
|x(\sigma(u))| \leq \int_{\sigma(u)}^{t_{1}} q(s)|x(\sigma(s))| d s .
$$


Moreover,

$$
\begin{aligned}
|x(\xi)| & =\left|x(\xi)-x\left(t_{1}\right)\right|=\left|\int_{t_{1}}^{\xi} x^{\prime}(u) d u\right| \\
& =\left|\int_{t_{1}}^{\xi} q(u) x(\sigma(u)) d u\right| \\
& \leq \int_{t_{1}}^{\xi} q(u)|x(\sigma(u))| d u,
\end{aligned}
$$

and

$$
\begin{aligned}
|x(\xi)| & =\left|x\left(t_{2}\right)-x(\xi)\right|=\left|\int_{\xi}^{t_{2}} x^{\prime}(u) d u\right| \\
& \leq \int_{\xi}^{t_{2}} q(u)|x(\sigma(u))| d u .
\end{aligned}
$$

Thus, we obtain

$$
|x(\xi)| \leq \frac{1}{2} \int_{t_{1}}^{t_{2}} q(u)|x(\sigma(u))| d u .
$$

Here we consider two cases.

Case 1: $Q(T)<1$. We note $\beta>0$. By (2.5) and (2.6),

$$
|x(\xi)| \leq \frac{1}{2} \int_{t_{1}}^{t_{2}} q(u) \int_{\sigma(u)}^{t_{1}} q(s)|x(\sigma(s))| d s d u .
$$

Since $|x(\xi)|>0$,

$$
\int_{t_{1}}^{t_{2}} q(u) \int_{\sigma(u)}^{t_{1}} q(s) d s d u>0 .
$$

By (2.4), we see that $|x(\sigma(s))|<K e^{-\beta h(\sigma(s))}$ for $s \in\left[\sigma^{-1}\left(t_{0}\right), t_{1}\right]$. Then, by (2.7) and (2.8),

$$
|x(\xi)|<\frac{1}{2} \int_{t_{1}}^{t_{2}} q(u) \int_{\sigma(u)}^{t_{1}} q(s) K e^{-\beta h(\sigma(s))} d s d u .
$$

Since $h(t)$ is increasing and $\beta>0$,

$$
\begin{aligned}
|x(\xi)| & <\frac{K}{2} \int_{t_{1}}^{t_{2}} q(u) e^{-\beta h\left(\sigma^{2}(u)\right)} \int_{\sigma(u)}^{t_{1}} q(s) d s d u \\
& \leq \frac{K}{2} e^{-\beta h\left(\sigma^{2}\left(t_{1}\right)\right)} \int_{t_{1}}^{t_{2}} q(u) \int_{\sigma(u)}^{t_{1}} q(s) d s d u .
\end{aligned}
$$


Then

$$
|x(\xi)|<\frac{K}{2} e^{-\beta h\left(\sigma^{2}\left(t_{1}\right)\right)}\left\{\int_{t_{1}}^{t_{2}} q(u) \int_{\sigma(u)}^{u} q(s) d s d u+\int_{t_{1}}^{t_{2}} q(u) \int_{u}^{t_{1}} q(s) d s d u\right\} .
$$

Since

$$
\begin{gathered}
\int_{t_{1}}^{t_{2}} q(u) \int_{u}^{t_{1}} q(s) d s d u=-\frac{1}{2}\left(\int_{t_{1}}^{t_{2}} q(u) d u\right)^{2}, \\
|x(\xi)|<\frac{K}{2} e^{-\beta h\left(\sigma^{2}\left(t_{1}\right)\right)}\left\{Q(T) \int_{t_{1}}^{t_{2}} q(u) d u-\frac{1}{2}\left(\int_{t_{1}}^{t_{2}} q(u) d u\right)^{2}\right\} .
\end{gathered}
$$

The right side of (2.9) is a quadratic function of $\int_{t_{1}}^{t_{2}} q(u) d u$ and $0<$ $\int_{t_{1}}^{t_{2}} q(u) d u \leq \int_{\sigma\left(t_{2}\right)}^{t_{2}} q(u) d u \leq Q(T)$. Then

$$
\begin{aligned}
|x(\xi)| & <\frac{K}{2} e^{-\beta h\left(\sigma^{2}\left(t_{1}\right)\right)}\left(Q^{2}(T)-\frac{1}{2} Q^{2}(T)\right) \\
& \leq \frac{Q^{2}(T) K}{4} e^{-\beta h\left(\sigma^{3}(\xi)\right)} \\
& \leq \frac{Q^{2}(T) K}{4} e^{\beta\left(h(\xi)-h\left(\sigma^{3}(\xi)\right)\right)} e^{-\beta h(\xi)}
\end{aligned}
$$

By (2.1),

$$
|x(\xi)|<\frac{Q^{2}(T)}{4} e^{3 \delta \beta}\left(K e^{-\beta h(\xi)}\right)
$$

Thus, (2.3) implies $|x(\xi)|<K e^{-\beta h(\xi)}$. Then we have a contradiction to the assumption that $|x(\xi)|=K e^{-\beta h(\xi)}$. bilities.

Case 2: $1 \leq Q(T) \leq 5 / 2$. We note $\beta \geq 0$. There are two possi-

Case 2.1: $1 \leq \int_{t_{1}}^{t_{2}} q(t) d t \leq 5 / 2$. There exists an $\eta$ such that $t_{1} \leq \eta<t_{2}$ and $\int_{\eta}^{t_{2}} q(t) d t=1$. By (2.5) and (2.6), we have

$$
\begin{aligned}
|x(\xi)| & \leq \frac{1}{2} \int_{t_{1}}^{t_{2}} q(u)|x(\sigma(u))| d u \\
& =\frac{1}{2} \int_{t_{1}}^{\eta} q(u)|x(\sigma(u))| d u+\frac{1}{2} \int_{\eta}^{t_{2}} q(u)|x(\sigma(u))| d u \\
& \leq \frac{1}{2} \int_{t_{1}}^{\eta} q(u)|x(\sigma(u))| d u+\frac{1}{2} \int_{\eta}^{t_{2}} q(u) \int_{\sigma(u)}^{t_{1}} q(s)|x(\sigma(s))| d s d u
\end{aligned}
$$




$$
\begin{aligned}
= & \frac{1}{2} \int_{t_{1}}^{\eta} q(u)|x(\sigma(u))| d u \\
& +\frac{1}{2} \int_{\eta}^{t_{2}} q(u)\left\{\int_{\sigma(u)}^{\eta} q(s)|x(\sigma(s))| d s+\int_{\eta}^{t_{1}} q(s)|x(\sigma(s))| d s\right\} d u .
\end{aligned}
$$

By $\int_{\eta}^{t_{2}} q(t) d t=1$,

$$
\int_{t_{1}}^{\eta} q(u)|x(\sigma(u))| d u=-\int_{\eta}^{t_{2}} q(u) \int_{\eta}^{t_{1}} q(s)|x(\sigma(s))| d s d u,
$$

which implies

$$
|x(\xi)| \leq \frac{1}{2} \int_{\eta}^{t_{2}} q(u) \int_{\sigma(u)}^{\eta} q(s)|x(\sigma(s))| d s d u .
$$

Since $|x(\xi)|>0$

$$
\int_{\eta}^{t_{2}} q(u) \int_{\sigma(u)}^{\eta} q(s) d s d u>0 .
$$

By (2.4) and the fact that $\sigma(s) \leq t_{1} \leq \xi$ for $s \in\left[\sigma^{-1}\left(t_{0}\right), t_{2}\right],|x(\sigma(s))|<K e^{-\beta h(\sigma(s))}$ for $s \in\left[\sigma^{-1}\left(t_{0}\right), \eta\right]$. Then, by $(2.10)$ and (2.11),

$$
\begin{aligned}
|x(\xi)| & <\frac{1}{2} \int_{\eta}^{t_{2}} q(u) \int_{\sigma(u)}^{\eta} q(s) K e^{-\beta h(\sigma(s))} d s d u \\
& \leq \frac{K}{2} \int_{\eta}^{t_{2}} q(u) e^{-\beta h\left(\sigma^{2}(u)\right)} \int_{\sigma(u)}^{\eta} q(s) d s d u \\
& \leq \frac{K}{2} e^{-\beta h\left(\sigma^{2}\left(t_{1}\right)\right)} \int_{\eta}^{t_{2}} q(u) \int_{\sigma(u)}^{\eta} q(s) d s d u \\
& =\frac{K}{2} e^{-\beta h\left(\sigma^{2}\left(t_{1}\right)\right)}\left\{\int_{\eta}^{t_{2}} q(u) \int_{\sigma(u)}^{u} q(s) d s d u+\int_{\eta}^{t_{2}} q(u) \int_{u}^{\eta} q(s) d s d u\right\} .
\end{aligned}
$$

Since

$$
\begin{gathered}
\int_{\eta}^{t_{2}} q(u) \int_{u}^{\eta} q(s) d s d u=-\frac{1}{2}\left(\int_{\eta}^{t_{2}} q(u) d u\right)^{2} \\
|x(\xi)|<\frac{K}{2} e^{-\beta h\left(\sigma^{2}\left(t_{1}\right)\right)}\left\{Q(T) \int_{\eta}^{t_{2}} q(u) d u-\frac{1}{2}\left(\int_{\eta}^{t_{2}} q(u) d u\right)^{2}\right\} \\
=\frac{K}{2} e^{-\beta h\left(\sigma^{2}\left(t_{1}\right)\right)}\left(Q(T) \cdot 1-\frac{1}{2} \cdot 1^{2}\right)
\end{gathered}
$$




$$
\begin{aligned}
& \leq \frac{2 Q(T)-1}{4} K e^{-\beta h\left(\sigma^{3}(\xi)\right)} \\
& =\frac{2 Q(T)-1}{4} e^{\beta\left(h(\xi)-h\left(\sigma^{3}(\xi)\right)\right)} K e^{-\beta h(\xi)} \\
& \leq \frac{2 Q(T)-1}{4} e^{3 \delta \beta}\left(K e^{-\beta h(\xi)}\right) .
\end{aligned}
$$

Thus, (2.3) implies $|x(\xi)|<K e^{-\beta h(\xi)}$. Then we have a contradiction to the assumption that $|x(\xi)|=K e^{-\beta h(\xi)}$.

Case 2.2: $\int_{t_{1}}^{t_{2}} q(t) d t<1$. In the same way as Case 1 , we have

$$
|x(\xi)|<\frac{K}{2} e^{-\beta h\left(\sigma^{2}\left(t_{1}\right)\right)}\left\{Q(T) \int_{t_{1}}^{t_{2}} q(u) d u-\frac{1}{2}\left(\int_{t_{1}}^{t_{2}} q(u) d u\right)^{2}\right\} .
$$

Since the right side of the above inequality is a quadratic function of $\int_{t_{1}}^{t_{2}} q(u) d u$ and $0<\int_{t_{1}}^{t_{2}} q(u) d u<1 \leq Q(T)$,

$$
\begin{aligned}
|x(\xi)| & <\frac{K}{2} e^{-\beta h\left(\sigma^{2}\left(t_{1}\right)\right)}\left(Q(T) \cdot 1-\frac{1}{2} \cdot 1^{2}\right) \\
& \leq \frac{2 Q(T)-1}{4} K e^{-\beta h\left(\sigma^{3}(\xi)\right)} \\
& =\frac{2 Q(T)-1}{4} e^{\beta\left(h(\xi)-h\left(\sigma^{3}(\xi)\right)\right)} K e^{-\beta h(\xi)} \\
& \leq \frac{2 Q(T)-1}{4} e^{3 \delta \beta}\left(K e^{-\beta h(\xi)}\right) .
\end{aligned}
$$

By (2.3), $|x(\xi)|<K e^{-\beta h(\xi)}$. Then we have a contradiction to the assumption that $|x(\xi)|=K e^{-\beta h(\xi)}$.

Hence, by virtue of the Case 1 and 2, we obtain (2.2). The proof is complete.

Using Theorem 1, we have two corollaries.

Corollary 1. If $Q(T) \leq 5 / 2$ for some $T \geq \sigma^{-1}\left(t_{0}\right)$, then every oscillatory solution of (1.1) is bounded.

Corollary 2. If $Q_{\infty}<5 / 2$, then every oscillatory solution of (1.1) tends to zero as $t \rightarrow \infty$.

Example 1. If $\sigma(t)=t-\tau(t)$ with a function $\tau(t)$ satisfying $0 \leq \tau(t) \leq \tau_{0}$ $\left(\tau_{0}>0\right)$, then we can take $h(t)=t$. Hence Theorem 1 shows that if $Q(T) \leq 5 / 2$ for some $T \geq \sigma^{-1}\left(t_{0}\right)$, then every oscillatory solution $x(t)$ of 
(1.1) satisfies

$$
|x(t)|<K e^{-\beta t}
$$

for $\beta$ given by (2.3) with $\delta=\tau_{0}$.

Essentially the same result was obtained by Györi ([2, Corollary 4.2]) under the condition $Q(T) \leq 2$ for some $T \geq \sigma^{-1}\left(t_{0}\right)$. So, our Theorem 1 is an improvement of Györi's result in this special case.

Example 2. In case $t_{0}>1 / a$ and $\sigma(t)=a t$ with $0<a<1$, we can take $h(t)=\log t$, so that $e^{-\beta h(t)}=t^{-\beta}$ in Theorem 1 .

Example 3. In case $t_{0}>e^{p}$ and $\sigma(t)=t^{p}$ with $0<p<1$, we can take $h(t)=\log (\log t)$, so that $e^{-\beta h(t)}=(\log t)^{-\beta}$ in Theorem 1 .

Remark. In the case where $5 / 2<Q(T)<\infty$ for some $T \geq \sigma^{-1}\left(t_{0}\right)$, we can show the following result in the same way as Case 1 in the proof of Theorem 1:

If there exists a continuous increasing function $h:\left[t_{0}, \infty\right) \rightarrow[0, \infty)$ such that $h(t)-h(\sigma(t)) \geq \delta_{1}$ for some constant $\delta_{1}>0$, then every oscillatory solution $x(t)$ of $(1.1)$ is estimated by

$$
|x(t)|<K e^{\alpha h(t)}
$$

where $K=K(\alpha, h, x)$ is a positive constant and $\alpha=\left(2 / \delta_{1}\right) \log (Q(T) / 2)$. For example, when $t-\sigma(t) \geq \delta_{1}>0$, we can take $h(t)=t$, so that $|x(t)|<K e^{\alpha t}$.

Now, we give a consequence of Corollary 2 .

Lemma 3. Let $x(t)$ be a solution of (1.1) and $Q_{\infty}<\infty$. If $x(t)$ is not oscillatory, then there exist a $T_{0}=T_{0}(x)>t_{0}$ and a positive constant $C=C(x)$ such that

$$
|x(t)| \geq C e^{\int_{t_{0}}^{t} q(u) d u /\left(Q_{\infty}+2\right)}, \quad t>T_{0} .
$$

Proof. Without loss of generality, we may assume that $x(t)$ is eventually positive, i.e., there exists $t_{1}>t_{0}$ such that $x(t)>0$ for any $t>t_{1}$. Choose $T_{0}>t_{0}$ such that $\sigma^{3}\left(T_{0}\right)>t_{1}$ and

$$
\int_{\sigma(t)}^{t} q(s) d s<Q_{\infty}+1, \quad t>T_{0} .
$$

By $(1.1)$ and $x(t) \neq 0$ for $t>t_{1}$,

$$
\int_{T_{0}}^{t} \frac{x^{\prime}(u)}{x(u)} d u=\int_{T_{0}}^{t} q(u) \frac{x(\sigma(u))}{x(u)} d u, \quad t>T_{0},
$$


which implies

$$
x(t)=x\left(T_{0}\right) e^{\int_{T_{0}}^{t} q(u) x(\sigma(u)) / x(u) d u} .
$$

Since $x^{\prime}(t) \geq 0$ for $t>\sigma^{-1}\left(t_{1}\right)$, we have

$$
\begin{aligned}
x(t)-x(\sigma(t)) & =\int_{\sigma(t)}^{t} x^{\prime}(u) d u \\
& =\int_{\sigma(t)}^{t} q(u) x(\sigma(u)) d u \\
& \leq x(\sigma(t)) \int_{\sigma(t)}^{t} q(u) d u \leq x(\sigma(t))\left(Q_{\infty}+1\right), \quad t>T_{0} .
\end{aligned}
$$

Then

$$
\frac{x(\sigma(t))}{x(t)} \geq \frac{1}{Q_{\infty}+2}
$$

which leads to that

$$
\begin{aligned}
x(t) & \geq x\left(T_{0}\right) e^{\int_{T_{0}}^{t} q(u) d u /\left(Q_{\infty}+2\right)} \\
& =x\left(T_{0}\right) e^{-\int_{t_{0}}^{T_{0}} q(u) d u /\left(Q_{\infty}+2\right)} e^{\int_{t_{0}}^{t} q(u) d u /\left(Q_{\infty}+2\right)}, \quad t>T_{0} .
\end{aligned}
$$

The proof is complete.

By this lemma we obtain the following theorem:

Theorem 2. Assume $Q_{\infty}<5 / 2$.

(i) If a solution $x(t)$ of (1.1) satisfies

$$
\lim _{t \rightarrow \infty} \frac{x(t)}{e^{\int_{t_{0}}^{t} q(u) d u /\left(Q_{\infty}+2\right)}}=0
$$

then $x(t)$ tends to zero as $t \rightarrow \infty$.

(ii) If

$$
\int_{t_{0}}^{\infty} q(u) d u=\infty
$$

then every bounded solution of (1.1) tends to zero as $t \rightarrow \infty$.

Proof. (i) By Lemma 3 and (2.12), $x(t)$ is oscillatory. Therefore, by Corollary 2, $x(t)$ tends to zero as $t \rightarrow \infty$.

(ii) Let $x(t)$ be a bounded solution of (1.1). By (2.13), $x(t)$ satisfies (2.12). Hence $x(t)$ tends to zero as $t \rightarrow \infty$.

The proof is complete. 


\section{Equations with special forcing term}

Let $A_{0}$ be the set of continuously differentiable functions $a(t)$ on $\left[\sigma\left(t_{0}\right), \infty\right)$ such that $\lim _{t \rightarrow \infty} a(t)=0$.

Consider the following equation:

$$
x^{\prime}(t)=q(t) x(\sigma(t))+f(t), \quad t \geq t_{0},
$$

where $f(t)$ is given as $f(t)=q(t) a(\sigma(t))-a^{\prime}(t)$ with some $a \in A_{0}$. We compare the asymptotic behavior of the oscillatory solution of (3.1) with that of the homogeneous equation (1.1).

Lemma 4. Let $y(t)$ be a continuous, piecewise continuously differentiable function on $\left[t_{0}, \infty\right)$ and $a \in A_{0}$. If $z(t)=y(t)+a(t)$ is a solution of (1.1) and $y(t)$ is oscillatory, then $z(t)$ is also oscillatory.

Proof. Assume that $z(t)$ is not oscillatory. Then there exists $t_{1}>\sigma^{-1}\left(t_{0}\right)$ such that $|z(t)|>0$ for $t>t_{1}$. Without loss of generality, we may assume that $z(t)>0$. Since $z(t)$ is a solution of $(1.1)$ and $q(t) \geq 0, z^{\prime}(t)=q(t) z(\sigma(t)) \geq 0$ for $t>t_{2}$ for some $t_{2}>\sigma^{-1}\left(t_{1}\right)$. Then $z(t) \geq z\left(t_{2}\right)>0$ for $t>t_{2}$. Since $a(t)$ tends to zero as $t \rightarrow \infty$, we have

$$
\liminf _{t \rightarrow \infty} y(t)=\liminf _{t \rightarrow \infty}\{z(t)-a(t)\} \geq z\left(t_{2}\right)>0 .
$$

This is a contradiction to the assumption that $y(t)$ is oscillatory. The proof is complete.

Theorem 3. If every oscillatory solution of (1.1) tends to zero as $t \rightarrow \infty$, then every oscillatory solution of (3.1) tends to zero as $t \rightarrow \infty$.

Proof. Let $x(t)$ be an oscillatory solution of (3.1). Then it follows that

$$
x^{\prime}(t)=q(t) x(\sigma(t))+q(t) a(\sigma(t))-a^{\prime}(t),
$$

which implies that $(x(t)+a(t))^{\prime}=q(t)(x(\sigma(t))+a(\sigma(t)))$. Since $z(t)=$ $x(t)+a(t)$ is a solution of (1.1), we see from Lemma 4 that $z(t)$ is oscillatory. Therefore $z(t)$ tends to zero as $t \rightarrow \infty$ by assumption. Hence $x(t)=z(t)-a(t)$ tends to zero as $t \rightarrow \infty$. The proof is complete.

Corollary 3. If $Q_{\infty}<5 / 2$, then every oscillatory solution of (3.1) tends to zero as $t \rightarrow \infty$.

Proof. By Corollary 2, every oscillatory solution of (1.1) tends to zero as $t \rightarrow \infty$. By Theorem 3, we obtain that every oscillatory solution of (3.1) tends to zero as $t \rightarrow \infty$. The proof is complete. 
Now, let us consider the equation:

$$
x^{\prime}(t)=q x(t-\tau)+k e^{-\lambda t}, \quad t \geq t_{0},
$$

where $k$ is a constant, $q, \tau$ and $\lambda$ are positive constants.

Theorem 4. Every oscillatory solution of (3.2) tends to zero as $t \rightarrow \infty$, if and only if every oscillatory solution of (1.3) tends to zero as $t \rightarrow \infty$.

Proof. Sufficiency. Suppose that every oscillatory solution of (1.3) tends to zero as $t \rightarrow \infty$. Define $a(t)$ as

$$
a(t)=\frac{k}{q e^{\lambda \tau}+\lambda} e^{-\lambda t} .
$$

Then we see that $a \in A_{0}$ and $k e^{-\lambda t}=q a(t-\tau)-a^{\prime}(t)$. By Theorem 3, every oscillatory solution of (3.2) tends to zero as $t \rightarrow \infty$.

Necessity. Suppose that every oscillatory solution of (3.2) tends to zero as $t \rightarrow \infty$ for some $k \neq 0$. Let $y(t)$ be an oscillatory solution of (1.3) and let $z(t)=y(t)-a(t)$. Then

$$
\begin{aligned}
z^{\prime}(t) & =q z(t-\tau)+q a(t-\tau)-a^{\prime}(t) \\
& =q z(t-\tau)+k e^{-\lambda t},
\end{aligned}
$$

which means that $z(t)$ is a solution of (3.2). We will prove that $z(t)$ tends to zero as $t \rightarrow \infty$. If $z(t)$ is oscillatory, then $z(t)$ tends to zero as $t \rightarrow \infty$ by assumption. Therefore it is enough to consider the case that $z(t)$ is not oscillatory. We will show that for some $T^{*}>t_{0}$,

$$
0<|z(t)| \leq \frac{|k|}{\lambda} e^{-\lambda t}, \quad t>T^{*}
$$

Let $k>0$. Assume $z(t)>0$ for $t>T_{1}$ for some $T_{1}>t_{0}$. Since $q z(t-\tau)>0$ for $t>T_{1}+\tau, z^{\prime}(t)>0$ for $t>T_{1}+\tau$. Then $z(t)$ is monotone increasing on $\left(T_{1}+\tau, \infty\right)$, which implies that for $T_{2}>T_{1}+\tau$,

$$
\begin{aligned}
\liminf _{t \rightarrow \infty} y(t) & \geq \liminf _{t \rightarrow \infty} z(t)+\liminf _{t \rightarrow \infty} a(t) \\
& \geq z\left(T_{2}\right)>0
\end{aligned}
$$

Since $y(t)$ is oscillatory, we have a contradiction. Hence $z(t)<0$ for $t>T_{3}$ for some $T_{3}>t_{0}$. Then

$$
\left(z(t)+\frac{k}{\lambda} e^{-\lambda t}\right)^{\prime}=q z(t-\tau)<0, \quad t>T_{3}+\tau,
$$


which implies that $z(t)+k e^{-\lambda t} / \lambda$ is monotone decreasing on $\left(T_{3}+\tau, \infty\right)$. Then we have that for any $u>T_{3}+\tau$,

$$
\begin{aligned}
\limsup _{t \rightarrow \infty} y(t) & =\limsup _{t \rightarrow \infty}(z(t)+a(t)) \\
& \leq \limsup _{t \rightarrow \infty}\left(z(t)+\frac{k}{\lambda} e^{-\lambda t}\right)+\limsup _{t \rightarrow \infty}\left(a(t)-\frac{k}{\lambda} e^{-\lambda t}\right) \\
& \leq\left(z(u)+\frac{k}{\lambda} e^{-\lambda u}\right) .
\end{aligned}
$$

Since $\lim \sup _{t \rightarrow \infty} y(t) \geq 0, z(u)+k e^{-\lambda u} / \lambda \geq 0$ for $u>T_{3}+\tau$. Therefore

$$
0>z(t) \geq-\frac{k}{\lambda} e^{-\lambda t}, \quad t>T_{3}+\tau
$$

In case $k<0$, we see in the same way that

$$
0<z(t) \leq-\frac{k}{\lambda} e^{-\lambda t}, \quad t>T_{4},
$$

for some $T_{4}>t_{0}$. Then we have (3.3), and hence $z(t)$ tends to zero as $t \rightarrow \infty$ when $z(t)$ is not oscillatory. Therefore $y(t)=z(t)+a(t)$ tends to zero as $t \rightarrow \infty$, which implies that every oscillatory solution of (1.3) tends to zero as $t \rightarrow \infty$. The proof is complete.

By Theorem 4 and Györi's result ([2, Theorem 4.2]), we have

Corollary 4. Every oscillatory solution of (3.2) tends to zero as $t \rightarrow \infty$, if and only if $q \tau<3 \pi / 2$.

\section{Example}

In this section, we consider an example which shows that $Q_{\infty}<15 / 4$ is a necessary condition for every oscillatory solution of (1.1) to tend to zero as $t \rightarrow \infty$. by

Let $q(t):[0, \infty) \rightarrow[0, \infty)$ be a piecewise continuous function defined

$$
q(t)= \begin{cases}k & (3 n \leq t<3 n+1+c) \\ 0 & (3 n+1+c \leq t<3(n+1))\end{cases}
$$

for $n=0,1,2, \ldots$, where $k=\alpha^{2}+5 \alpha / 8$ and $c=\alpha / 2 k$ for $\alpha \geq 1$ are constants.

Consider the delay differential equation:

$$
x^{\prime}(t)=q(t) x(t-4), \quad t \geq 0,
$$


with initial function $\phi$ such that

$$
\phi(t)= \begin{cases}-1 & (-4 \leq t \leq-3) \\ \frac{k}{\alpha}(t+3)-1 & (-3<t<-3+c) \\ \frac{2 \alpha+1}{2(2-c)}(t+1)+\alpha & (-3+c \leq t \leq-1) \\ \alpha & (-1 \leq t \leq 0) .\end{cases}
$$

Then we see that $x(t)$ is given by

$$
x(t)=(-\alpha)^{n} \begin{cases}\alpha-k(t-3 n) & (3 n \leq t<3 n+1) \\ \alpha-k(t-3 n)+\frac{k^{2}}{2 \alpha}(t-3 n-1)^{2} & (3 n+1 \leq t<3 n+1+c) \\ -\alpha^{2} & (3 n+1+c \leq t<3(n+1))\end{cases}
$$

for $n=0,1,2, \ldots$ Therefore it follows that

(a) if $\alpha>1$, then $k>13 / 8, c<4 / 13$, lim $\sup _{t \rightarrow \infty}|x(t)|=\infty$ and

$$
\limsup _{t \rightarrow \infty} \int_{t-4}^{t} q(s) d s=k(2+c)=2 \alpha^{2}+\frac{7}{4} \alpha>\frac{15}{4},
$$

(b) if $\alpha=1$, then $k=13 / 8, c=4 / 13, \lim \sup _{t \rightarrow \infty}|x(t)|=1$ and

$$
\limsup _{t \rightarrow \infty} \int_{t-4}^{t} q(s) d s=\frac{15}{4} .
$$

This fact shows that there exists an oscillatory solution of (1.1) which does not tend to zero as $t \rightarrow \infty$ when

$$
\limsup _{t \rightarrow \infty} \int_{t-4}^{t} q(s) d s \geq \frac{15}{4} .
$$

Acknowledgement. The author would sincerely like to thank the anonymous referee for his/her careful reading and valuable comments to revise this manuscript, and Professor Fumi-yuki Maeda for his many useful suggestions and kind support which help me to improve this paper.

\section{References}

[1] Erbe, L. H., Kong, Qingkai and Zhang, B. G., Oscillation theory for functional differential equations, Monographs and Textbooks in Pure and Applied Mathematics, 190, Marcel Dekker, Inc., New York, 1995.

[2] Györi, I., Existence and growth of oscillatory solutions of first order unstable type delay differential equations, Nonlinear Anal., 13 (1989), 739-751. 
[3] Kuang, Y., Delay Differential Equations with Applications in Population Dynamics, Mathematics in Science and Engineering, 191, Academic Press, Inc., Boston, MA, 1993.

[ 4 ] Ladas, G. and Sficas, Y. G., Asymptotic behavior of oscillatory solutions, Hiroshima Math. J. 18 (1988), 351-359.

[5] Ladde, G. S., Lakshmikantham, V. and Zhang, B. G., Oscillation Theory of Differential Equations with Deviating Arguments, Monographs and Textbooks in Pure and Applied Mathematics, 110, Marcel Dekker, Inc., New York, 1987.

[6] Matsunaga, H., Miyazaki, R. and Hara, T., Global attractivity results for nonlinear delay differential equations, J. Math. Anal. Appl., 234 (1999), 77-90.

[7] Muroya, Y., On Yoneyama's $3 / 2$ stability theorems for one-dimensional delay differential equations, J. Math. Anal. Appl., 247 (2000), 314-322.

[ 8 ] Philos, Ch. G., Purnaras, I. and Sficas, Y. G., Asymptotic Decay of the Oscillatory Solutions to First Order Non-Autonomous Linear Unstable Type Delay Differential Equations, Funkcial. Ekvac., 49 (2006), 385-413.

[9] Yoneyama, T., On the $3 / 2$ stability theorem for one-dimensional delay-differential equations, J. Math. Anal. Appl., 125 (1987), 161-173.

[10] Yoneyama, T., The $3 / 2$ stability theorem for one-dimensional delay-differential equations with unbounded delay, J. Math. Anal. Appl., 165 (1992), 133-143.

\author{
nuna adreso: \\ Obirin High School \\ Tokiwa 3758, Machida \\ Tokyo 194-0294 \\ Japan \\ E-mail: tat235711@ac.auone-net.jp
}

(Ricevita la 31-an de januaro, 2012)

(Reviziita la 30-an de januaro, 2013) 